\title{
Machine and labor flexibility in manufacturing networks
}

\author{
David Francas ${ }^{\mathrm{a}, *}$, Nils Löhndorf ${ }^{\mathrm{b}}$, Stefan Minner ${ }^{\mathrm{b}}$ \\ a Department of Logistics, University of Mannheim, Germany \\ ${ }^{\mathrm{b}}$ Department of Business Administration, University of Vienna, Austria
}

\section{A R T I C L E I N F O}

\section{Article history:}

Received 26 June 2008

Accepted 16 March 2010

\section{Keywords:}

Machine and labor flexibility

Manufacturing networks

Stochastic programming

\begin{abstract}
A B S T R A C T
Many industrial companies today operate under strict employment legislation and work agreements. Since flexible capacity has been widely recognized as an important hedge against uncertain demand, there exist strong efforts to introduce more flexible workforce models. In this paper, we investigate the benefits of such labor flexibility and its interplay with machine flexibility from a network capacity investment perspective.

We consider a firm that has to invest simultaneously in labor and machine capacity under uncertain demand and a given network configuration while anticipating the deployment of labor flexibility after demand has been realized. Instruments of labor flexibility range from temporary employment to personnel transfers between plants. The underlying decision problem is formulated as a two-stage stochastic program with recourse.

Based on numerical studies and the analysis of a stylized model, we demonstrate the impact of labor flexibility on the optimal levels of machine and labor capacity. We compare the benefits obtained by personnel transfers with those of temporary workers and find that temporary employment always decreases the number of permanent workers, while personnel transfers may even allow for a larger workforce. Our results further indicate that personnel transfers are more effective in larger manufacturing networks although these benefits are decreasing when most plants in the network are capable of producing more than one product (machine flexibility). Finally, we present evidence for the efficiency of a combined usage of personnel transfers and temporary workers.
\end{abstract}

(c) 2010 Elsevier B.V. All rights reserved.

\section{Introduction}

In the past decades, competition has intensified and customers are more demanding than ever before. Increasing product variety and shorter product life cycles have accelerated demand uncertainty, and the development of instruments which attain a better match of supply and demand has become a key ingredient to maintain sufficient capacity utilization and high service levels. Capital-intensive industries such as car or semiconductor manufacturing (Suarez et al., 1996) encounter difficulties to adjust their capacities in the short run. Furthermore, strict employment legislation, work agreements, and no-lay-off policies lead to long-term commitments in workforce, as illustrated in Askar et al. (2007) and Jack and Raturi (2002). For these reasons, improving flexibility is widely regarded as a crucial element of an effective manufacturing strategy.

The automotive industry is notoriously exposed to the risk of low capacity utilization, and a variety of measures have been taken to improve flexibility (Francas et al., 2009). The recent change in Renault's manufacturing strategy demonstrates the

\footnotetext{
* Corresponding author. Tel.: +496211811651.

E-mail address: francas@bwl.uni-mannheim.de (D. Francas).
}

new paradigm, away from inflexible one-plant/one-vehicle policies towards highly flexible machines and manufacturing platforms that are capable of producing multiple products. Renault's manufacturing chief Michel Gornet argued in face of this change that "since demand can be unpredictable, industrial strategy must integrate flexibility" (De Saint-Seine, 2007)

Also labor, as an essential resource and major cost factor, has received increasing attention. Managers from the automotive industry refer to labor flexibility as the plant's way to breathe because it is often the only alternative to adjust capacity in the short run. In face of the global financial crisis of 2008 and its negative impact on automotive sales it is flexibility that helps BMW to avoid layoffs in their US plants (Barkholz, 2009). For instance, Daimler's initiative "Safeguarding the Future: 2012" (Zukunftssicherung 2012) has been launched to improve competitiveness of the German sites and includes several measures to make the workforce more flexible. The measures allow Daimler to employ eight percent of its workers as temporary employees. Furthermore, trade unions and work councils agreed that all new employees and graduated apprentices enter a flexible workforce pool called "DC Move". All members of this pool can be transferred between German plants whenever necessary (Daimler, 2005).

However, developing a comprehensive flexibility strategy is not a simple task in manufacturing networks. Concerning the 
multi-dimensional and complex nature of manufacturing flexibility, operations managers would greatly benefit from insights about relationships, trade-offs, and synergies between different flexibility dimensions (Vokurka and O'Leary-Kelly, 2000). In particular, the relationship between machine and labor flexibility has been rarely covered in network design models although its importance is emphasized by practitioners and several empirical studies (Askar et al., 2007; Jack and Raturi, 2002). Both sources of flexibility constitute elements of mix and volume flexibility which are recognized as the most crucial flexibility dimensions. Mix flexibility entails the ability to use one resource to build multiple products, while volume flexibility accounts for the ability to ramp output volume up or down without incurring large cost (Goyal and Netessine, 2009).

In this paper, we analyze a planning model to provide insights into the interplay of labor and machine flexibility from a capacity investment perspective. We investigate the dependencies between strategic investments in machine and labor capacity, flexible network structures, and labor flexibility instruments such as personnel transfers and temporary workers.

The remainder of the paper is organized as follows. After a review of related literature in Section 2 we develop a two-stage stochastic optimization model for the network design problem with operational labor flexibility (Section 3). In Section 4 we analyze a two-plant, two-product version of this model and subsequently present numerical studies for the general case with multiple plants (Section 5). Finally, in Section 6 we conclude with a summary and discussion of our findings and give an outlook on future research.

\section{Literature review}

We focus on literature on mix and volume flexibility with emphasis on capacity investment models. For detailed reviews on manufacturing flexibility we refer to Gerwin (1993), Koste and Malhotra (1999), and Bertrand (2003). In particular, Koste and Malhotra (1999) provide a theoretical framework for the hierarchy of different flexibility dimensions and classify labor and machine flexibility as lower-level dimensions of mix and volume flexibility.

Our paper investigates machine and labor flexibility from a strategic planning perspective and thus, a detailed discussion of different labor instruments and their modeling is omitted. We refer to Pinker and Larson (2003) for a detailed review, and to Askar et al. (2007) for modeling labor flexibility in the automotive industry.

The standard model of mix flexibility is a single-period, multiproduct, two-stage stochastic program with random demand where the first-stage decisions consist of investments in dedicated and flexible resources. At the second stage, the production quantities are determined after uncertainty about demand has been resolved. In their seminal work, Fine and Freund (1990) investigate this problem for an $n$ product firm that invests in $n$ dedicated resources and one flexible resource which is capable of producing all products. The authors show how these investments depend on the cost difference between dedicated and flexible technologies. Van Mieghem (1998) investigates a variation of this model and shows that mix flexibility can even be beneficial under perfectly positively correlated demand if product margins vary. Van Mieghem and Rudi (2002) introduce "newsvendor networks" as a general framework to study such problems of stochastic capacity investment.

Partially flexible systems are treated in Jordan and Graves (1995). They show that there exist network configurations with limited flexibility which yield nearly the same expected profits as fully flexible systems and develop the chaining principle as a guideline for designing such systems. The benefits of limited resource flexibility are studied in Aksin et al. (2005). As in our model, they control the degree of mix flexibility through an exogenous parameter that restricts capacity available to other products.

Our model of sharing labor resources through personnel transfers is also related to the investment in commonality studied by Kulkarni et al. (2005) and Lu and Van Mieghem (2009). Similar to common components in a production process, the ability to transfer workers implies that labor capacity is input to several plants. Formally, such commonality is an equivalent formulation of the mix-flexibility theme (Van Mieghem, 2004).

The second dimension of flexibility investigated in this paper is volume flexibility. An empirical analysis of its sources and its linkage to mix flexibility can be found in Jack and Raturi (2002). Surprisingly, model-based research on volume-flexible technology and capacity investment is rare. In planning models for the automotive industry, Chandra et al. (2005) and Fleischmann et al. (2006) model volume flexibility through the usage of overtime. Their notion of overtime is similar to our notion of temporary workers as it allows to increase initial capacity levels at the second stage.

The relationship between volume and mix flexibility is investigated by Goyal and Netessine (2009). They consider a two-product firm which faces random demand with products being either substitutes or complements and system configuration being either dedicated or fully flexible. Their notion of volume flexibility is that capacity levels can be adjusted up or down at quadratic cost after demand has been realized. They show how cost and demand parameters affect the optimal technology choice and demonstrate that adding mix flexibility to volume flexibility may provide only limited advantages if capacity adjustment costs are convex. In particular, they demonstrate that mix flexibility better mitigates uncertainty in demand for individual products than volume flexibility, while volume flexibility better handles uncertainty in aggregate demand. Finally, Chod et al. (2007) study mix, volume, and time flexibility and show that mix and volume flexibility act as substitutes but are both complementary to time flexibility.

Although its importance is emphasized by practitioners and several empirical studies (Askar et al., 2007; Jack and Raturi, 2002), the interplay between labor and machine flexibility has not yet been studied from a strategic capacity perspective. Our model allows to analyze this interplay and extends the work of Aksin et al. (2005) by incorporating limited volume flexibility and capacity choice. We also address the interplay between mix and volume flexibility. Contrary to the work of Goyal and Netessine (2009), we study investments in two different capacity types with different flexibility options: mix-flexible machine capacity and limited mix- and volume-flexible labor capacity.

\section{Model}

\subsection{Assumptions}

Supply chain model: Consider a firm which builds $m$ products in $n$ plants. Demand for each product is uncertain and modeled by a multi-variate random vector $\boldsymbol{D} \in \mathbb{R}_{+}^{m}$ with known probability distribution. In face of uncertain demand, the firm decides on a strategic investment in labor and machine capacities, $\boldsymbol{K}^{\boldsymbol{L A}} \in \mathbb{R}_{+}^{n}$ and $\boldsymbol{K}^{\boldsymbol{M A}} \in \mathbb{R}_{+}^{n}$. After investments are made and demand is realized, the firm optimally allocates these resources to product demands. The firm is modeled as a price-taker, unsatisfied demand is lost, and all costs are linear functions. In line with 
literature (e.g., Fine and Freund, 1990; Van Mieghem, 1998; Goyal and Netessine, 2009), variable production costs are normalized to zero and shifting production between plants induces no additional variable cost. The network configuration is exogenously given by a matrix $A$ which denotes the assignment of products to plants (and hence the degree of machine flexibility), i.e., if product $i$ can be manufactured in plant $j$ then $a_{i j}=1$, otherwise $a_{i j}=0$. Manufacturing one unit of product $i$ in plant $j$ consumes one unit of machine capacity in plant $j$.

Labor flexibility instruments: Labor flexibility instruments are aligned along two flexibility dimensions. Instruments of volume flexibility, such as temporary employment and paid overtime, are labeled as temporary workers, and instruments of mix flexibility, such as job rotation schemes and inter-plant transfers, are referred to as personnel transfers. It is assumed that the maximum usage of labor instruments is constrained by exogenously given parameters and available workforce, which is similar to the model formulations in Chandra et al. (2005) and Aksin et al. (2005). A parameter $\alpha \in[0,1]$ is introduced that restricts the percentage of a plant's workforce which can be transferred to other plants. In a similar fashion, parameter $\beta \in[0, \infty)$ restricts the maximum amount of temporary workers that can be hired in each plant in addition to regular workforce. These structural parameters reflect organizational capabilities, employment legislation, or work agreements. It is further assumed that all plants are located relatively close to each other (e.g., consider the production network of a German car manufacturer), and thus labor transfers are allowed between all plants. Lastly, manufacturing one unit of product $i$ in plant $j$ consumes one unit of regular or flexible labor in plant $j$.

\subsection{Model formulation}

The network design problem is modeled as a single-period, two-stage stochastic program. The first-stage objective $V$ represents the expected value of the maximization problem (1)-(8) with $\Pi$ as the second-stage objective function. The objective in (1) is to maximize the difference of expected second-stage profits $\mathbb{E}(\Pi)$ and first-stage investment costs for capacities $K_{j}^{M A}$ and $K_{j}^{L A}$ (with marginal cost $c_{j}^{M A}$ and $c_{j}^{L A}$ ). The maximum value of $V(\cdot)$ is denoted by $V^{*}$ while $\boldsymbol{K}^{L A^{*}}$ and $\boldsymbol{K}^{\boldsymbol{M} \boldsymbol{A}^{*}}$ represent the optimal capacity acquisition vectors. The expectation $\mathbb{E}_{\boldsymbol{D}}$ is taken with respect to the random demand vector $\boldsymbol{D}$ :

$V^{*}\left(\boldsymbol{K}^{\mathbf{L} \boldsymbol{A}^{*}}, \boldsymbol{K}^{\boldsymbol{M} \boldsymbol{A}^{*}}\right)=\max _{\boldsymbol{K}^{\mathbf{L} A}, \boldsymbol{K}^{M A} \in \mathbb{R}_{+}^{n}}\left[\mathbb{E}_{\boldsymbol{D}}(\Pi)-\sum_{j=1}^{n}\left(c_{j}^{L A} K_{j}^{L A}+c_{j}^{M A} K_{j}^{M A}\right)\right]$.

The second stage is formulated as a product-mix problem. Let $p_{i}$ denote the sales price of product $i, c_{j}^{t w}$ the costs of temporary workers in plant $j$, and $c_{j k}^{t r}$ the per unit costs of personnel transfers between plants $j$ and $k$. For a given realization of product demand $d_{i}$, the firm chooses production quantities $x_{i j}$ and the usage of temporary workers $t w_{j}$ and personnel transfers $t r_{j k}$ to maximize its operational profit $\Pi$.

$\Pi=\max _{\boldsymbol{x}, \boldsymbol{t r}, \boldsymbol{t w}}\left[\sum_{i=1}^{m} \sum_{j=1}^{n} p_{i} x_{i j}-\sum_{j=1}^{n} \sum_{k=1}^{n} c_{j k}^{t r} t r_{j k}-\sum_{j=1}^{n} c_{j}^{t w} t w_{j}\right]$

with $\boldsymbol{x} \in \mathbb{R}_{+}^{m \times n}, \boldsymbol{t r} \in \mathbb{R}_{+}^{n \times n}, \boldsymbol{t w} \in \mathbb{R}_{+}^{n}$

s.t. $\quad \sum_{k=1}^{n} t r_{j k} \leq \alpha K_{j}^{L A} \quad \forall j=1, \ldots, n$

$t w_{j} \leq \beta K_{j}^{L A} \quad \forall j=1, \ldots, n$

$$
\begin{aligned}
& \sum_{i=1}^{m} x_{i j} \leq K_{j}^{L A}+t w_{j}+\sum_{k=1}^{n}\left(t r_{k j}-t r_{j k}\right) \quad \forall j=1, \ldots, n \\
& \sum_{i=1}^{m} x_{i j} \leq K_{j}^{M A} \quad \forall j=1, \ldots, n \\
& x_{i j} \leq a_{i j} K_{j}^{M A} \quad \forall i=1, \ldots, m, j=1, \ldots, n \\
& \sum_{j=1}^{n} x_{i j} \leq d_{i} \quad \forall i=1, \ldots, m .
\end{aligned}
$$

Personnel transfers and temporary workers are constrained by (3) and (4) as their usage may not exceed the degree of flexibility, $\alpha$ and $\beta$, inherent in a plant's workforce $K_{j}^{L A}$. Production in each plant is constrained by (5) to available labor capacity, which is the sum of regular workforce, temporary workers, and personnel transfers; it is further constrained by available machine capacity through (6). The allocation of products to plants is constrained in (7) by the network configuration $A$, which defines the degree of machine flexibility. Finally, production is restricted by demand $d_{i}$ in (8).

\section{Model analysis}

\subsection{General model}

Increasing labor flexibility usually requires longsome negotiations with trade unions and work councils as well as costly organizational changes. For these reasons, the benefits of more flexibility should be carefully evaluated. The impact of changes in the structural parameters $\alpha$ and $\beta$ on the optimal objective value $V^{*}$, and thus, the benefits that can be obtained by increasing the degree of labor flexibility, is shown through the following property.

Property 1. The optimal value $V^{*}$ is increasing and concave in $\alpha$ and $\beta$.

The proof is given in the Appendix. The marginal benefit of either type of labor flexibility is decreasing, which indicates declining scale earnings. Property 1 generalizes Property 1 in Aksin et al. (2005), who obtain this result for exogenously given capacity levels.

\subsection{Stylized model}

Before presenting numerical results for the general model (1)(8), a simplified two-plant, two-product model is studied to obtain some insights into the optimal choice of the two capacity types, labor and machine capacity, when only temporary workers or personnel transfers are available. Furthermore, it is studied how the deployment of these two labor flexibility instruments depends on network configuration, price and cost parameters, demand correlation, and demand volatility of the two products. Each labor flexibility instrument is analyzed separately for a fully flexible $\left(A=\left(\begin{array}{ll}1 & 1 \\ 1 & 1\end{array}\right)\right)$ and a dedicated $\left(A=\left(\begin{array}{ll}1 & 0 \\ 0 & 1\end{array}\right)\right)$ configuration of machine capacity.

A two-point discrete demand distribution is assumed for this purpose. The coefficient of correlation is denoted by $\rho$. We consider the cases $\rho=-1$ and 1 . If $\rho=1$, the demand realizations are $(1+\hat{\sigma}, 1+\hat{\sigma})$ and $(1-\hat{\sigma}, 1-\hat{\sigma})$, each with probability $\frac{1}{2}$, where $\hat{\sigma} \in(0,1)$ is a measure of demand volatility. If $\rho=-1$, realizations are $(1+\hat{\sigma}, 1-\hat{\sigma})$ and $(1-\hat{\sigma}, 1+\hat{\sigma})$, each with probability $\frac{1}{2}$. In the following, we denote $1-\hat{\sigma}$ as low demand scenario, while $1+\hat{\sigma}$ is referred to as high demand scenario. Furthermore, symmetric cost 
and price parameters are assumed, i.e., $p=p_{1}=p_{2}, c^{M A}=c_{1}^{M A}=c_{2}^{M A}$, $c^{L A}=c_{1}^{L A}=c_{2}^{L A}$. The costs for temporary workers $c^{t w}$ and personnel transfers $c^{t r}$ are identical in both plants. By setting $p_{1}=p_{2}$, we exclude the revenue maximization option as further advantage of flexible network configurations (see Van Mieghem, 1998; Lu and Van Mieghem, 2009). All cost and price parameters are assumed to be greater than zero.

We make the following assumptions regarding the economic parameters.

C1 $p>c^{L A}+c^{M A}$ : It is economically justified to produce both products regardless of labor flexibility at hand.

C2 $c^{t w}>c^{L A}$ : The use of temporary workers or overtime is more expensive than hiring workers on a regular basis.

C3 $c^{t w}<2 c^{L A}$ and $c^{t r}<2 c^{L A}$ : Expected costs for labor flexibility instruments do not exceed costs for direct investment in labor capacity.

Finally, let $\alpha, \beta>0$ to enable personnel transfers and temporary workers, respectively. Otherwise, trivial solutions would be obtained since labor flexibility instruments would be unavailable.

Before stating the special cases of temporary workers only and personnel transfers only, we state a property of an optimal solution in the dedicated network which is exploited for the derivation of the following results.

Property 2. Optimal machine and labor capacity levels in plant $j$ satisfy in the dedicated network: $(1-\hat{\sigma}) \leq K_{j}^{L A}{ }^{*} \leq K_{j}^{M A^{*}} \leq 1+\hat{\sigma}$.

Proof. Assumption $p>c^{L A}+c^{M A}$ ensures that satisfying the certain component of product demand $(1-\hat{\sigma})$ is always profitable, while $c^{t w}>c^{L A}$ and $c^{t r}>0$ favor direct investment in labor capacity over flexible instruments for processing this certain base demand. The upper inequality holds since production in a plant cannot exceed demand. If the middle inequality does not hold, excess labor capacity cannot be used in plant $j$. In case of labor transfers, a redeployment of capacity is more efficient since $c_{1}^{L A}=c_{2}^{L A}$ and $c^{\text {tr }}>0$.

The derivations of the subsequent results can be found in the Appendix.

\subsubsection{Temporary workers $(\alpha=0)$}

The optimal decisions depend on the degree of flexibility and the products' profitability. Therefore, we define the conditions $F L^{T}$ and $F H^{T}$ to denote the degree of flexibility through temporary workers and $P L^{T}, P M^{T}$, and $P H^{T}$ to distinguish the firm's profitability with respect to price and cost parameters.

$$
\begin{aligned}
& F L^{T} \quad \text { Flexibility low } \quad \beta<\frac{2 \hat{\sigma}}{1-\hat{\sigma}} \\
& F H^{T} \text { Flexibility high } \beta \geq \frac{2 \hat{\sigma}}{1-\hat{\sigma}} \\
& P L^{T} \quad \text { Low } \\
& p<2 c^{M A}+c^{t w} \\
& 2 c^{M A}+c^{t w} \leq p<c^{t w} \frac{\beta}{(1+\beta)}+2 c^{M A}+\frac{2 c^{L A}}{(1+\beta)} \\
& p \geq c^{t w} \frac{\beta}{(1+\beta)}+2 c^{M A}+\frac{2 c^{L A}}{(1+\beta)}
\end{aligned}
$$

Four cases (i)-(iv) are distinguished with respect to the different values of coefficient $\rho$ and network configuration $A$. Table 1 shows the optimal capacity levels and the expected deployment of labor flexibility. For each case, the optimal aggregate capacity levels $\sum_{j=1}^{2} K_{j}^{L A^{*}}$ and $\sum_{j=1}^{2} K_{j}^{M A^{*}}$ and expected temporary worker usage $\mathbb{E} \sum_{j=1}^{2} t w_{j}$ are shown in the columns of the table.

Cases (i) and (ii): If condition $P L^{T}$ holds, it is not beneficial to increase capacity above $\sum_{j=1}^{2} K_{j}^{L A^{*}}=\sum_{j=1}^{2} K_{j}^{M A^{*}}=2(1-\hat{\sigma})$ since the use of temporary workers is too costly. In this case, only the fraction of demand $1-\hat{\sigma}$ that is certain is satisfied in each plant since it is too costly to buy capacity for the uncertain fraction of demand $(2 \hat{\sigma})$.

If conditions $P M^{T}$ and $F L^{T}$ hold, it is beneficial to use temporary workers in the high demand scenarios. However, the sales price is not high enough to justify hiring workforce which would only be utilized in the high demand scenarios. Thus $\sum_{j=1}^{2} K_{j}^{L A^{*}}=2(1-\hat{\sigma})$. Furthermore, the condition $F L^{T}$ implies that if demand is high it is not possible to satisfy additional labor requirements by use of temporary workers. As a consequence, the aggregate capacity is set to $\sum_{j=1}^{2} K_{j}^{M A^{*}}=2(1-\hat{\sigma})(1+\beta)$, which allows full exploitation of available labor flexibility $\beta(1-\hat{\sigma})$ in each plant.

If conditions $P H^{T}$ and $F L^{T}$ hold, expected profits are high enough to justify investments in regular workforce which will only be used in the high demand scenario. In this case, each plant's labor and machine capacity is set to a level that ensures that demand is always met. Hence, $\sum_{j=1}^{2} K_{j}^{M A^{*}}=2(1+\hat{\sigma})$, labor investments are $\sum_{j=1}^{2} K_{j}^{L A^{*}}=2(1+\hat{\sigma}) /(1+\beta)$, and if demand is high the remaining demand for labor is met by using temporary workers.

Table 1

Optimal capacity acquisition and expected usage of temporary workers.

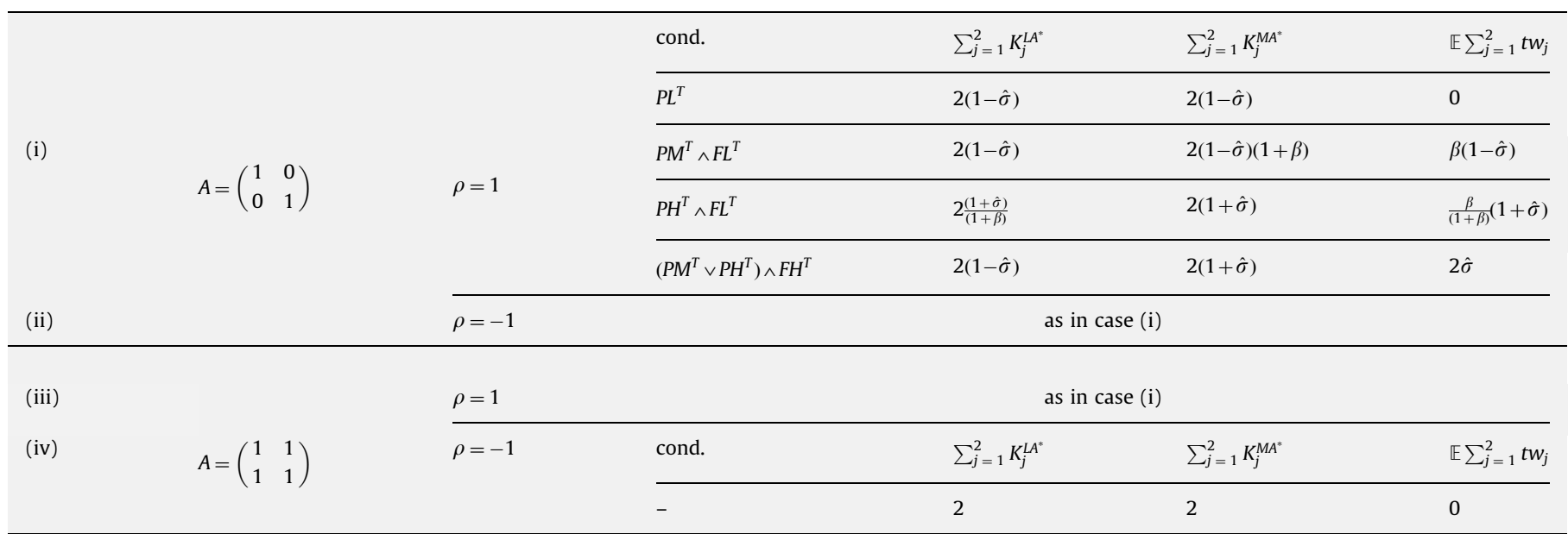


If $P M^{T}$ or $P H^{T}$ holds and condition $F H^{T}$ is satisfied, temporary workers are profitable and can entirely satisfy additional needs for labor in high demand scenarios. Hence, $\sum_{j=1}^{2} K_{j}^{L A^{*}}=2(1-\hat{\sigma})$ and $\sum_{j=1}^{2} K_{j}^{M A^{*}}=2(1+\hat{\sigma})$.

Case (iii): Demand pooling is not possible in the fully flexible network $A=\left(\begin{array}{ll}1 & 1 \\ 1 & 1\end{array}\right)$ if demands are perfectly positively correlated. Then, production cannot be shifted from a product with low demand to a product with high demand since demand moves in lockstep. Similar to the dedicated configurations (cases (i) and (ii)), temporary workers have to be used to mitigate demand uncertainty. Therefore, we obtain the same decisions as in cases (i) and (ii).

Case (iv): In the fully flexible network $A=\left(\begin{array}{ll}1 & 1 \\ 1 & 1\end{array}\right)$ with negative demand correlation, temporary workers are not used to mitigate demand uncertainty. Instead, production is shifted from the product with low demand to the product with high demand (or equivalently, the firm invests in only one plant since identical investment costs are assumed). Therefore, temporary workers are substituted by the (costless) option to shift production by means of machine flexibility. Thus, aggregate capacity is set to the aggregate demand level (which is identical in both scenarios), $\sum_{j=1}^{2} K_{j}^{L A^{*}}=\sum_{j=1}^{2} K_{j}^{M A^{*}}=2$.

In summary, the results show that temporary workers (as a type of volume flexibility) are used regardless of the sign of correlation ( $\rho=-1$ or 1 ), which is in line with the results of Goyal and Netessine (2009). However, there exists a trade-off between machine and labor flexibility. Less surprisingly, depending on the ratio between sales price $p$ and strategic and operational costs, demand volatility $\hat{\sigma}$ can increase and decrease capacity acquisition and the usage of temporary workers. Flexibility through temporary workers allows to adjust capacity when needed and thus enables lower investment in regular workforce. Moreover, it allows to increase overall system capacity since the risk of low capacity utilization is reduced.

\subsubsection{Personnel transfers $(\beta=0)$}

In line with the previous analysis, we distinguish four cases with respect to the different choices of demand correlation $\rho$ and network configuration $A$, labeled (i)-(iv). The optimal decisions depend on the investment cost level $C H^{P}$ or $C L^{P}$, the degree of flexibility $F L^{P}$ or $F H^{P}$, and the product profitability $P L^{P}$, $P M^{P}, P H^{P}$.

$$
\begin{array}{lll}
C H^{P} & \begin{array}{l}
\text { High } \\
\text { investment } \\
\text { costs }
\end{array} & p<2 c^{M A}+2 c^{L A} \\
C L^{P} & \begin{array}{l}
\text { Low } \\
\text { investment } \\
\text { costs } \\
F L^{P}
\end{array} & \begin{array}{l}
\text { Low } \\
\text { flexibility }
\end{array} \quad \alpha<\hat{\sigma} \\
F H^{P} & \begin{array}{l}
\text { High } \\
\text { flexibility }
\end{array} \quad \alpha \geq \hat{\sigma} \\
P L^{P} & \begin{array}{l}
\text { Low } \\
\text { profitability }
\end{array} \\
P M^{P} & \begin{array}{l}
\text { Medium } \\
\text { profitability }
\end{array} \\
P H^{P} & \begin{array}{l}
\text { High } \\
\text { profitability }
\end{array} & p \leq c^{M A}+c^{L A}+\frac{c^{L A}}{(1+\alpha)}+\frac{c^{t r}}{2} \leq p<c^{t r} \frac{\alpha}{(1+\alpha)}+2 c^{M A}+\frac{2 c^{L A}}{(1+\alpha)} \\
&
\end{array}
$$

Table 2 shows the optimal network capacity levels as well as expected personnel transfers.

Cases (i) and (iii): When demands are perfectly positively correlated, the firm neither benefits from production shifting nor from labor transfers (as types of mix flexibility) because product demands move in lockstep. If $C L^{P}$ holds, investment costs for labor and machines capacity are low enough to cover the expected revenues of the uncertain demand component in the high demand scenarios. Then, capacity is set to its maximum and $\sum_{j=1}^{2} K_{j}^{L A^{*}}=\sum_{j=1}^{2} K_{j}^{M A^{*}}=2(1+\hat{\sigma})$.

\begin{tabular}{|c|c|c|c|c|c|c|}
\hline \multirow{3}{*}{ (i) } & \multirow{8}{*}{$A=\left(\begin{array}{ll}1 & 0 \\
0 & 1\end{array}\right)$} & \multirow{3}{*}{$\rho=1$} & cond. & $\sum_{j=1}^{2} K_{j}^{L A^{*}}$ & $\sum_{j=1}^{2} K_{j}^{M A^{*}}$ & $\mathbb{E} \sum_{j=1}^{2} \sum_{k=1}^{2} \operatorname{tr}_{j k}$ \\
\hline & & & $\mathrm{CH}^{P}$ & $2(1-\hat{\sigma})$ & $2(1-\hat{\sigma})$ & 0 \\
\hline & & & $C L^{P}$ & $2(1+\hat{\sigma})$ & $2(1+\hat{\sigma})$ & 0 \\
\hline \multirow{5}{*}{ (ii) } & & \multirow{5}{*}{$\rho=-1$} & cond. & $\sum_{j=1}^{2} K_{j}^{L A^{*}}$ & $\sum_{j=1}^{2} K_{j}^{M A^{*}}$ & $\mathbb{E} \sum_{j=1}^{2} \sum_{k=1}^{2} t r_{j k}$ \\
\hline & & & $P L^{P}$ & $2(1-\hat{\sigma})$ & $2(1-\hat{\sigma})$ & 0 \\
\hline & & & $P M^{P} \wedge F L^{P}$ & $2 \frac{(1-\hat{\sigma})}{(1-\alpha)}$ & $2(1+\alpha) \frac{(1-\hat{\sigma})}{(1-\alpha)}$ & $\frac{\alpha}{(1-\alpha)}(1-\hat{\sigma})$ \\
\hline & & & $P H^{P} \wedge F L^{P}$ & $2 \frac{(1+\hat{\sigma})}{(1+\alpha)}$ & $2(1+\hat{\sigma})$ & $\frac{\alpha}{(1+\alpha)}(1+\hat{\sigma})$ \\
\hline & & & $\left(P M^{P} \vee P H^{P}\right) \wedge F H^{P}$ & 2 & $2(1+\hat{\sigma})$ & $\hat{\sigma}$ \\
\hline
\end{tabular}

Case (ii): If condition $P L^{P}$ holds, it is not beneficial to increase capacity above $\sum_{j=1}^{2} K_{j}^{L A^{*}}=\sum_{j=1}^{2} K_{j}^{M A^{*}}=2(1-\hat{\sigma})$ since the use of personnel transfers is too costly. Only the fraction of demand which is certain $(1-\hat{\sigma})$ is produced in each plant, because it is too costly to also buy capacity for the uncertain fraction of demand $(2 \hat{\sigma})$.

If conditions $P M^{P}$ and $F L^{P}$ hold, it is beneficial to use personnel transfers in the high demand scenarios of the two products. In contrast to the usage of temporary workers, the deployment of personnel transfers requires workforce levels higher than the base demand $1-\hat{\sigma}$ in each plant. Otherwise, workforce would be fully

Table 2

Optimal capacity acquisition and expected personnel transfers.

(iii)

(iv) $\rho=1$

$$
A=\left(\begin{array}{ll}
1 & 1 \\
1 & 1
\end{array}\right)
$$

$\rho=-1$ as in case (i)

cond.

$\sum_{j=1}^{2} K_{j}^{L \mu}$

$\sum_{j=1}^{2} K_{j}^{M A}$

$\mathbb{E} \sum_{j=1}^{2} \sum_{k=1}^{2} t r_{j k}$ 
utilized with producing the base demand $1-\hat{\sigma}$ in each scenario and would not be available for transfers to the other plant. Furthermore, $F L^{P}$ implies that parameter $\alpha$ is restricting personnel transfers, and thus, not all permanently employed workers can be transferred to the other plant if needed. As a consequence, regular workers are hired as long as they can be used for personnel transfers to the other plant. This results in aggregate capacity levels $\sum_{j=1}^{2} K_{j}^{L A^{*}}=2(1-\hat{\sigma}) /(1-\alpha)<2$ and $\sum_{j=1}^{2} K_{j}^{M A^{*}}=2(1+\alpha)$ $(1-\hat{\sigma}) /(1-\alpha)<2(1+\hat{\sigma})$.

If conditions $P H^{P}$ and $F L^{P}$ hold, expected profits are high enough to justify investments in regular workforce although only a fraction of workforce can be used for transfers in the high demand scenarios. In this case, each plant's labor and machine capacity is set to a level that ensures that demand can always be met. Therefore, $\sum_{j=1}^{2} K_{j}^{M A^{*}}=2(1+\hat{\sigma})$ and the regular workforce is set to $\sum_{j=1}^{2} K_{j}^{L A}=2(1+\hat{\sigma}) /(1+\alpha)$, while remaining labor requirements are met by transfers from the other plant if demand is high.

If $P M^{P}$ or $P H^{P}$ holds and the condition $F H^{P}$ is satisfied, personnel transfers are profitable and the structural parameter $\alpha$ does not restrict their deployment. An aggregated workforce level $\sum_{j=1}^{2} K_{j}^{L A^{*}}=2$ is sufficient to fully satisfy demand in the high demand scenarios. Hence, maximum machine capacity $\sum_{j=1}^{2} K_{j}^{M A^{*}}=2(1+\hat{\sigma})$ is required. A comparison of the different workforce levels $\sum_{j=1}^{2} K_{j}^{L A^{*}}$ in case (ii) shows that $2(1-\hat{\sigma}) /(1-\alpha) \leq 2 \leq 2(1+\hat{\sigma}) /(1+\alpha)$, so that the high availability of personnel transfers can have two effects. First, it serves as an incentive to increase regular workforce because the risk of underutilization is reduced $\left(F L^{P} \wedge P M^{P}\right.$ vs. $\left.F H^{P} \wedge P M^{P}\right)$. Second, it leads to lower investments due to the pooling effect $\left(F L^{P} \wedge P H^{P}\right.$ vs. $\left.F H^{P} \wedge P H^{P}\right)$.

Case (iv): In the fully flexible network $A=\left(\begin{array}{ll}1 & 1 \\ 1 & 1\end{array}\right)$ with negative demand correlation, personnel transfers are not used to mitigate demand uncertainty. Similar to case (iv) for temporary workers, using machine flexibility is less expensive than relying on flexible workforce models. Therefore, aggregate capacity is set $\sum_{j=1}^{2} K_{j}^{L A^{*}}=\sum_{j=1}^{2} K_{j}^{M A^{*}}=2$.

In contrast to temporary workers, the results indicate that the usage of personnel transfers in a two-plant setting crucially hinges on demand correlation, which limits its applicability. A trade-off between network flexibility and labor transfers is also observed. This substitution is likely to be more pronounced than with temporary workers since both production shifting and personnel transfers are types of mix flexibility. Since production shifting is assumed to induce no additional cost, it is preferred towards personnel transfers to balance capacities in the network.

In summary, both labor flexibility instruments provide an opportunity to reduce the risk of low capacity utilization by deploying a flexible workforce instead of hiring permanent staff. As the overall system capacity is determined by machine capacity, the firm needs to exercise its labor flexibility option to use its full capacity. Moreover, due to higher profitability compared to nonflexible firms, the presence of labor flexibility provides an incentive to increase machine and even permanent labor capacity.

\section{Numerical results}

\subsection{Experimental design}

The analysis in the previous section illustrated that each labor flexibility instrument depends on network configuration, price and cost parameters, as well as demand characteristics, and showed how labor flexibility influences optimal capacity acquisitions.
In order to study the impact of a simultaneous usage of both, temporary workers and personnel transfers, the model (1)-(8) is investigated numerically using a multi-variate normal distribution. The demand characteristics and prices of all products are assumed to be identical, and thus, the index $i$ is dropped in the following. As a consequence, identical sales prices $p$ across products exclude revenue maximization effects from the numerical analysis and allow to isolate the impact of different demand characteristics. The normal distribution is specified by mean $\mu$ and standard deviation $\sigma$ for all products and identical coefficient of correlation $\rho$ between demand for product $i$ and $l$ with $i \neq l$. In a similar fashion, it is assumed that all parameters are identical across plants and consequently, the index $j$ is dropped as well. This implies, in particular, that cost differentials are excluded as significant but obvious drivers of investment strategies and flexibility usage, and we focus on the rather subtle effects of the various flexibility enablers.

We use parameters $c^{L A}=c^{M A}=1, c^{t w}=1.2, c^{t r}=0.2, \alpha=\beta=0.5$, $p=4 \mu=100, \sigma=40$ and $\rho=-0.5$ as base case. Unless otherwise noted, a dedicated network configuration is assumed (e.g., $A=\left(\begin{array}{ll}1 & 0 \\ 0 & 1\end{array}\right)$ in case of a two-plant, two-product network). In order to measure the benefits of labor flexibility, we define the flexibility premium as

$\Delta F=\frac{V-V_{\alpha, \beta=0}}{V_{\alpha, \beta=0}}$

where $V_{\alpha, \beta}=0$ denotes the expected value of the profit maximization problem when no labor flexibility is available.

Demand is approximated via 10,000 pseudo-random scenarios. In order to avoid negative demand values the normal distribution is assumed to be truncated ( \pm 2.5 standard deviations). With this approximation, the optimization problem (1)-(8) can be rewritten as deterministic equivalent which is a linear program. We refer to Francas et al. (2009) for further details about this approximation approach.

\subsection{Benefit of temporary workers and personnel transfers}

First, it is examined how an increase in labor flexibility affects the total expected profit of the firm. By varying the structural parameters $\alpha$ and $\beta$ ceteris paribus, the degree of temporary workers and personnel transfers is incrementally adjusted and their effectiveness can be observed.

Fig. 1 shows a contour plot of the flexibility premium $\Delta F$ for $\alpha, \beta \in[0,0.5]$. The increasing distance of the contour lines indicates

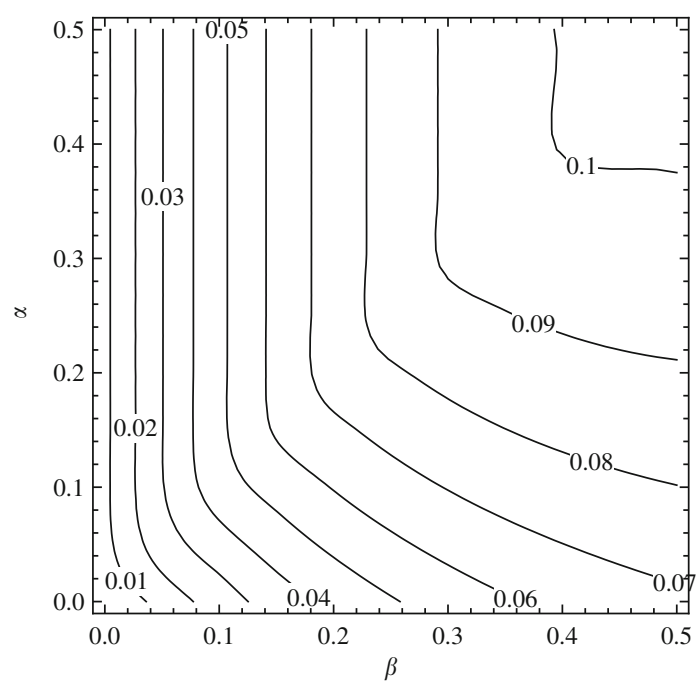

Fig. 1. Flexibility premium $\Delta F$ as a function of labor flexibility. 
that the marginal benefit of labor flexibility is decreasing, which illustrates the finding stated in Property 1 that expected profits increase as a concave function of $\alpha$ or $\beta$. For positive $\alpha$ the contour lines are closer together than for $\alpha=0.0$, which indicates that the marginal flexibility premium of temporary workers is higher for firms which use transfers than for firms that do not use transfers at all. Consequently, firms that allow personnel transfers may benefit more from temporary workers than firms that do not. If the contour lines are regarded as indifference curves, it can be seen that the marginal rate of substitution is infinity for $\alpha>\beta$ and nearly constant for $\alpha<\beta$, which indicates that both types of flexibility can be complements as well as substitutes. A firm always benefits from more temporary workers, but not always from more personnel transfers. For high levels of flexibility through personnel transfers, volume and mix flexibility behave like strategic complements. For any given level of flexibility through temporary workers, on the other hand, volume and mix flexibility behave like strategic substitutes.

\subsection{Synergies between temporary workers and transfers}

In Section 4.2, personnel transfers and temporary workers were analyzed separately, and their sensitivity to demand correlation was investigated using a two-point demand distribution. In order to further refine these results and investigate synergies between temporary workers and personnel transfers, we vary the correlation coefficient and examine its impact on the efficiency of each flexibility dimension.

Fig. 2 plots the flexibility premium $\Delta F$ for $\rho \in[-1,1]$. While the profitability of personnel transfers tapers off quickly as demand correlation increases, the influence of temporary workers remains unaffected by correlation, which is in line with our previous analysis and the results in Goyal and Netessine (2009). Moreover, the flexibility premium of a combination of both instruments is higher than the sum of the flexibility premium of personnel transfers and temporary workers if $\rho>-0.9$. This suggests the existence of synergies among the two flexibility types.

First, a higher flexibility premium can be explained by the fact that the response to individual demand scenarios is more costefficient with both instruments at hand. In case of scarce labor capacity in one plant and underutilized regular workforce in the other (high demand in one plant, low demand in the other), the less expensive reallocation of workers is preferred over the use of temporary workers (recall that $c^{t r}<c^{t w}$ ). On the other hand, temporary workers are required if labor capacity in both plants is insufficient (high demand in both plants).

Second, both instruments in conjunction also allow for a smart combination of both instruments' advantages. Suppose a firm faces

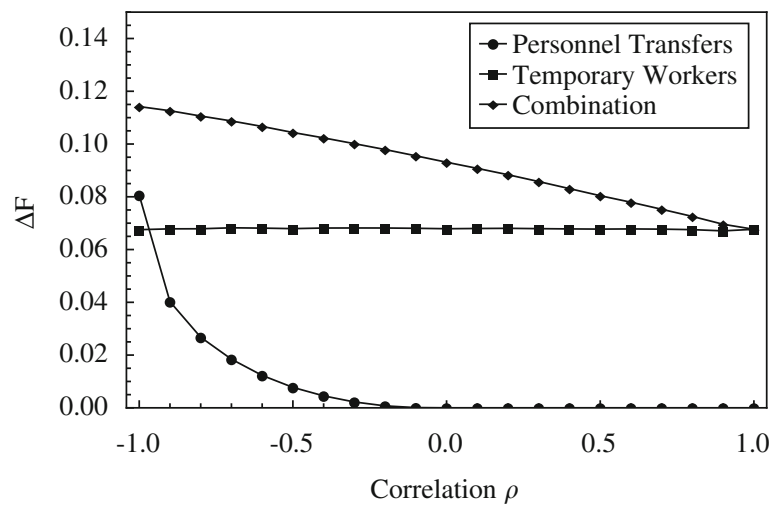

Fig. 2. Impact of temporary workers and personnel transfers on the flexibility premium as a function of demand correlation. aggregate demand higher than total labor capacity and one plant's workforce is not fully utilized. With both types of flexibility at hand, the firm can use temporary workers to increase capacity and transfer labor to rebalance capacity between plants. Let us illustrate this interplay with a small example. Consider a firm that only uses temporary workers in addition to its regular workforce. It employs 10 workers in its two plants which are paid 1 and but it needs to pay 2 for an additional temporary worker. In case demand is 5 in plant 1 and 20 in plant 2, plant 2 uses 10 temporary workers. Suppose the firm now has the additional option to transfer its personnel at half the cost of labor. The firm then transfers 5 workers from plant 1 to plant 2 and bridges the remaining gap with temporary workers. Consequently, the combination enables a more efficient use of labor. In particular, personnel transfers allow to balance the workload and thus increase labor utilization.

\subsection{Personnel transfers in networks with more than two plants}

In Section 4.2 it was argued that personnel transfers may not be used in a two-plant, two-product network with full machine flexibility. In the following, the benefit obtained by transfers within larger networks and other types of network configurations is investigated. In order to study the individual benefit of personnel transfers, temporary workers $(\beta=0)$ are excluded. With regard to Jordan and Graves (1995), partially flexible networks configured as chains and networks where pairs of plants are flexible in addition to dedicated configurations are studied. For instance, in a fourplant, four-product setting the corresponding entries of $A$ are

\begin{tabular}{c} 
Dedicated \\
$A=\left(\begin{array}{llll}1 & 0 & 0 & 0 \\
0 & 1 & 0 & 0 \\
0 & 0 & 1 & 0 \\
0 & 0 & 0 & 1\end{array}\right) \quad A=\left(\begin{array}{llll}1 & 1 & 0 & 0 \\
0 & 1 & 1 & 0 \\
0 & 0 & 1 & 1 \\
1 & 0 & 0 & 1\end{array}\right) \quad A=\left(\begin{array}{llll}1 & 1 & 0 & 0 \\
1 & 1 & 0 & 0 \\
0 & 0 & 1 & 1 \\
0 & 0 & 1 & 1\end{array}\right)$ \\
\hline
\end{tabular}

Fig. 3 plots the flexibility premium $\Delta F$ for network sizes $m \in\{2, \ldots, 10\}$ with $n=m$. The correlation coefficient is now reset to $\rho=0$.

First, we can observe that the benefits of personnel transfers diminish considerably once machine flexibility is introduced into the network. When all plants are linked together, as in the case of chaining, personnel transfers are not used at all. As long as a costless shifting of production volumes between plants, identical sales prices, and symmetric investment cost are assumed, workers are not transferred between flexible plants. A similar observation can be made for the configuration with flexible pairs if two or four

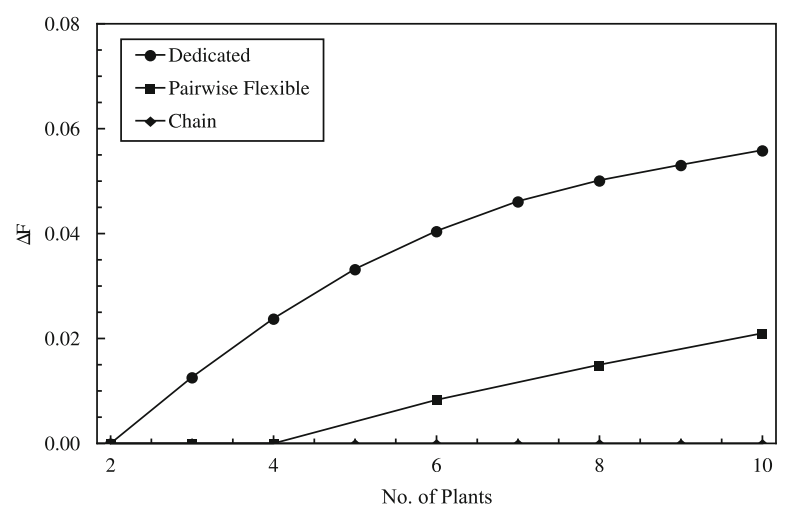

Fig. 3. Impact of network type on the flexibility premium as a function of network size. 
plants are considered. This observation corresponds with the analytical results from the stylized model.

In a dedicated or pairwise flexible network, personnel transfers yield a positive flexibility premium. For larger numbers of $n$, we observe a concave increasing flexibility premium which indicates a distinct pooling effect, in line with the findings of Eppen (1979). Once the number of plants increases, the probability of a capacity mismatch in one plant of the network rises. In larger networks, the overall quantity of available transfers increases and can be used to redistribute workforce more efficiently. Consequently, the firm invests less in its labor resources but uses transfers to pool its workforce instead.

\section{Conclusion}

We have studied the interrelations of investments in labor and machine resources and the role of different flexibility measures in production networks under uncertain demand. Based on a twostage stochastic programming approach, we have presented a model that accounts for temporary workers (overtime) as instruments of operational volume flexibility and personnel transfers (job rotation) as instrument of operational mix flexibility as well as flexible configurations of machine capacities.

Our analytical results indicate that the usage of labor flexibility instruments is a complex interplay of demand characteristics, price and cost parameters, as well as network configuration and structural parameters such as organizational capabilities or legal restrictions. We showed that the marginal benefit of both types of labor flexibility is decreasing. The analysis of a stylized model revealed that temporary workers always decrease investments in permanent staff. Personnel transfers, on the other hand, may lead to higher investments in regular workforce, because the additional flexibility has a positive influence on labor utilization.

Our numerical studies revealed that personnel transfers increase the efficiency of temporary workers, because there exist synergies between both types of flexibility. The results indicate that volume and mix flexibility can be both strategic complements as well as strategic substitutes, depending on the degree of flexibility. Consequently, a firm may benefit more from a good mix of both instruments than from an exclusive use of a single type of labor flexibility. The numerical results further showed that worker transfers may be more beneficial in larger networks, but that a flexible network or positive demand correlation can significantly reduce these benefits. In contrast, the usage of overtime is not sensitive to demand correlation and only partly substituted by flexible network structures.

Further research should address the development of planning tools that jointly capture labor and machine capacity and their flexibility enablers in more detail. In particular, relaxing the assumption of a costless production allocation by considering changeover costs could create additional trade-offs between machine and labor flexibility.

\section{Acknowledgments}

The authors acknowledge the input from Markus Friese of Daimler AG in helping provide context and direction for this research. The paper also benefited from the comments of Holger Stephan and Mirko Kremer.

\section{Appendix A}

Proof of Property 1. From the theory of linear programming, the value of a linear maximization program is concave in the coefficients of the right hand side (Bradley et al., 1977, pp. 694699). Thus, for a given demand realization and a given capacity level, $\Pi(\alpha)$ is concave in $\alpha$. Concavity is preserved under expectation, so $V\left(\boldsymbol{K}^{L A}, \boldsymbol{K}^{M A}, \alpha\right)$ is increasing and concave in $\alpha$ for given $\boldsymbol{K}^{\boldsymbol{L A}}$ and $\boldsymbol{K}^{\boldsymbol{M A}}$. Now let $\boldsymbol{K}^{\boldsymbol{L} \boldsymbol{A}^{*}}(\alpha)$ and $\boldsymbol{K}^{\boldsymbol{M} \boldsymbol{A}^{*}}(\alpha)$ be the optimal values of $\boldsymbol{K}^{\mathbf{L A}}$ and $\boldsymbol{K}^{\mathbf{M A}}$, expressed in terms of the structural parameter $\alpha$, so that $V^{*}(\alpha)=V^{*}\left(\boldsymbol{K}^{L A^{*}}(\alpha), \boldsymbol{K}^{M A^{*}}(\alpha), \alpha\right)$. The envelope

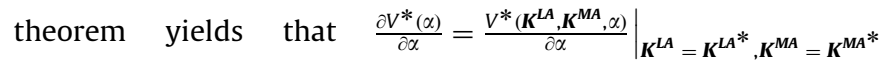
(Sydsæter and Hammond, 2002, p. 496). The proof for $\beta$ is analogous.

\section{A.1. Analysis of the stylized model}

Since the stochastic program in the stylized model is linear, its optimal first-stage solutions can be obtained by cost-revenue comparisons. Note that the optimal solutions are not necessarily unique and the allocation of capacity between the two plants is arbitrary in some cases. However, the aggregated capacity decisions are unique.

\section{A.2. Temporary workers}

Cases (i) and (ii): In the dedicated network, the decisions in each plant can be analyzed separately.

Because of Property 2, we only derive the conditions that determine how much capacity is provided for the uncertain component of demand in the high demand scenario. Each unit of machine capacity above $K_{j}^{M A}=(1-\hat{\sigma})$ yields an expected sales price $\frac{1}{2} p$ in $j$ if we assume that labor requirements can be met. Since $c^{t w}<2 c^{L A}$, it is less costly to use temporary workers in order to meet labor requirements in the high demand scenarios.

1. Suppose that the uncertain component of demand is met by temporary workers (and $K_{j}^{L A^{*}}=1-\hat{\sigma}$ ). We invest in machine capacity if

$\frac{1}{2}\left(p-c^{t w}\right) \geq c^{M A}$.

Eq. (9) separates $P L^{T}$ and $P M^{T}$. Next, it has to be investigated when constraint (4), which restricts the available temporary workers, becomes binding.

1a. If

$(1-\hat{\sigma})(1+\beta)<(1+\hat{\sigma})$

holds, regular workforce $1-\hat{\sigma}$ plus available temporary workers $\beta(1-\hat{\sigma})$ are not sufficient to fully satisfy demand $1+\hat{\sigma}$ in the high demand scenarios. Eq. (10) separates $F L^{T}$ and $F H^{T}$. If $F L^{T}$ and $P M^{T}$ hold, (4) becomes binding if $\beta(1-\hat{\sigma})$ temporary workers are used. Thus, $K_{j}^{M A^{*}}=(1-\hat{\sigma})(1+\beta)$.

1b. If $F H^{T}$ and $\left(P M^{T}\right.$ or $\left.P H^{T}\right)$ hold, the uncertain component of demand can be completely produced by using temporary workers. Thus, $K_{j}^{M A^{*}}=(1+\hat{\sigma})$.

2. Next, the conditions for setting $K_{j}^{L A}>1-\hat{\sigma}$ are derived.

Suppose that $F L^{T}$ holds and $K_{j}^{M A}=(1-\hat{\sigma})(1+\beta)$. Satisfying one additional unit of demand in the high demand scenario requires one additional unit of $K_{j}^{M A}$ and one unit of additional labor capacity (either regular or temporary workforce). With respect to (4), the required units of additional regular workforce $\Delta K_{j}^{L A}=1 /(1+\beta)$ are given by $\Delta K_{j}^{L A}+\beta \Delta K_{j}^{L A}=1$, where $\beta \Delta K_{j}^{L A}$ denotes temporary worker usage. Thus, the use of temporary workers yields expected costs $\frac{1}{2}(\beta /(1+\beta)) c^{t w}$. Comparing 
(expected) costs with expected revenues yields

$\frac{1}{2} p \geq \frac{1}{2} \frac{\beta}{1+\beta} c^{t w}+c^{M A}+\frac{1}{(1+\beta)} c^{L A}$.

Eq. (11) separates $P M^{T}$ and $P H^{T}$. It is beneficial to fully satisfy demand in the high demand scenario and we obtain $K_{j}^{M A^{*}}=1+\hat{\sigma}$ and $K_{j}^{L A^{*}}=(1+\hat{\sigma}) /(1+\beta)$.

Cases (iii) and (iv) have already been treated in the text.

\section{A.3. Personnel transfers}

Case (i): If

$\frac{1}{2} p \geq c^{L A}+c^{M A}$

the expected revenue in the high demand scenario exceeds investment costs. Eq. (12) separates $C L^{P}$ and $C H^{P}$. If $C L^{P}$ holds, maximum capacity is acquired and $\sum_{j=1}^{2} K_{j}^{L A^{*}}=\sum_{j=1}^{2}$ $K_{j}^{M A^{*}}=2(1+\hat{\sigma})$.

Case (ii): We reformulate the stochastic program (1)-(8) as follows:

$$
\begin{aligned}
V= & -c^{L A}\left(K_{1}^{L A}+K_{2}^{L A}\right)-c^{M A}\left(K_{1}^{M A}+K_{2}^{M A}\right)+\sum_{\omega=1}^{2} \frac{1}{2}\left(\left(p-c^{t r}\right)\left(x_{1 \omega}^{t r}+x_{2 \omega}^{t r}\right)\right. \\
& \left.+p\left(x_{1 \omega}+x_{2 \omega}\right)\right)
\end{aligned}
$$

s.t. $\quad x_{1 \omega}+x_{2 \omega}^{t r} \leq K_{1}^{L A} \quad \forall \omega=1,2$

$x_{2 \omega}+x_{1 \omega}^{t r} \leq K_{2}^{L A} \quad \forall \omega=1,2$

$x_{1 \omega}+x_{1 \omega}^{\mathrm{tr}} \leq K_{1}^{\mathrm{MA}} \quad \forall \omega=1,2$

$x_{2 \omega}+x_{2 \omega}^{t r} \leq K_{2}^{M A} \quad \forall \omega=1,2$

$x_{1 \omega}+x_{1 \omega}^{\operatorname{tr}} \leq d_{1 \omega} \quad \forall \omega=1,2$

$x_{2 \omega}+x_{2 \omega}^{t r} \leq d_{2 \omega} \quad \forall \omega=1,2$

$x_{1 \omega}^{t r} \leq \alpha K_{2}^{L A} \quad \forall \omega=1,2$

$x_{2 \omega}^{t r} \leq \alpha K_{1}^{L A} \quad \forall \omega=1,2$

$K_{1}^{L A}, K_{2}^{L A}, K_{1}^{M A}, K_{2}^{M A}, x_{1 \omega}, x_{2 \omega}, x_{1 \omega}^{t r}, x_{2 \omega}^{t r} \geq 0 \quad \forall \omega=1,2$

where $x_{i \omega}^{\text {tr }}$ denotes production of good $i$ using personnel transfers and $x_{i \omega}$ regular production.

The optimal second-stage policy is given by

$\boldsymbol{x}^{*}=\left(\begin{array}{cc}K_{1}^{L A} & 1-\hat{\sigma} \\ 1-\hat{\sigma} & K_{2}^{L A}\end{array}\right)$

$\boldsymbol{x}^{t r^{*}}=\left(\begin{array}{cc}\min \left\{\alpha K_{2}^{L A}, K_{2}^{L A}-(1-\hat{\sigma}), K_{1}^{M A}-K_{1}^{L A}\right\} & 0 \\ 0 & \min \left\{\alpha K_{1}^{L A}, K_{1}^{L A}-(1-\hat{\sigma}), K_{2}^{M A}-K_{2}^{L A}\right\}\end{array}\right)$

where rows refer to $i$ and columns refer to $\omega$.

In (24), $\min \left(\alpha K_{j}^{L A}, K_{j}^{L A}-(1-\hat{\sigma})\right)$ denotes amount of labor capacity in $j$ that can be used to manufacture product $i$, where $i, j=1,2 \mid i \neq j$. In particular, $\alpha K_{j}^{L A}$ denotes the available quantity if (20) or (21), respectively, is binding, while $K_{j}^{L A}-(1-\hat{\sigma})$ denotes the transferable labor capacity after $(1-\hat{\sigma})$ demand units have been satisfied in $j$. Furthermore, $K_{j}^{M A}-K_{j}^{L A}$ denotes the demand for labor capacity in $j$ to produce $i$, where $i, j=1,2 \mid i=j$.
In order to facilitate the analysis of case (i), we make use of the following property:

Property 3. An optimal capacity policy has the (sufficient but not necessary) property $K_{1}^{M A^{*}}=K_{2}^{M A^{*}}$ and $K_{1}^{L A^{*}}=K_{2}^{L A^{*}}$.

Note that the optimal solution is not necessarily unique but we can always find an optimal solution with this property.

Proof. Suppose we allocate a certain amount of capacity $K^{L A^{\prime}}=K_{1}^{L A^{\prime}}+K_{2}^{L A^{\prime}}$ and $K^{M A^{\prime}}=K_{1}^{M A^{\prime}}+K_{2}^{M A^{\prime}}$ with $K_{j}^{L A^{\prime}} \leq K_{j}^{M A^{\prime}}$ since $K_{j}^{L A^{*}} \leq K_{j}^{M A^{*}}, j=1,2$. In (13), $c^{L A}\left(K_{1}^{L A^{\prime}}+K_{2}^{L A^{\prime}}\right)+c^{M A}\left(K_{1}^{M A^{\prime}}+K_{2}^{M A^{\prime}}\right)$ and $\mathbb{E}\left[\sum_{i=1}^{2} x_{i}\right]=1-\hat{\sigma}+\frac{1}{2}\left(K_{1}^{L A^{\prime}}+K_{2}^{L A^{\prime}}\right)$ are not affected by the allocation. $K_{1}^{L A^{*}}=K_{2}^{L A^{*}}$ is a maximizer for the available transfers $\sum_{j=1}^{2} \min \left(\alpha K_{j}^{L A^{\prime}}, K_{j}^{L A^{\prime}}-(1-\hat{\sigma})\right)$. The aggregated demand for transfers $\sum_{j=1}^{2}\left(K_{j}^{M A^{\prime}}-K_{j}^{L A^{\prime}}\right)$ is not affected by the allocation since $K_{j}^{L A^{\prime}} \leq K_{j}^{M A^{\prime}}$. Thus, $K_{1}^{M A^{*}}=K_{2}^{M A^{*}}$ and $K_{1}^{L *^{*}}=K_{2}^{L A^{*}}$ is a maximizer for $\mathbb{E}\left[\sum_{i=1}^{2} x_{i}^{t r}\right]$.

We now derive the conditions that determine how much capacity is provided for the uncertain component of demand in the high demand scenarios.

1. Increasing expected production above $2(1-\hat{\sigma})$ by one unit in both plants yields $p$ (one unit of each product $i$ in its high demand scenario with probability $\frac{1}{2}$ ) and requires one unit of $K_{j}^{M A}$ in $j=1,2$. If (20) and (21) are not restricting transfers, an additional unit of $K_{k}^{L A}$ can be fully used for transfers to $j$ when demand in $k$ is low. Hence, only $\frac{1}{2} K_{j}^{L A}$ are needed since the missing $\frac{1}{2}$ units in $j$ are covered by $k$ resulting in transfer costs $\frac{1}{2} c^{\text {tr }}$ in the respective scenario. This yields

$p \geq 2 c^{M A}+c^{L A}+\frac{1}{2} c^{t r}$

as condition to invest in additional capacity. Eq. (25) separates $P L^{P}$ and $P M^{P}$.

1 a. To fully satisfy demand $1+\hat{\sigma}$ in the high demand scenarios of each product, $1-\hat{\sigma}+\hat{\sigma}$ units of labor capacity are needed in each plant $j$, while the remaining $\hat{\sigma}$ units can be provided by the other plant $k$ via personnel transfers if (20) and (21) are not restricting transfers. If

$\alpha(1-\hat{\sigma}+\hat{\sigma})<\hat{\sigma}$

holds, less than $\hat{\sigma}$ units can be transferred due to (20) and (21). Eq. (26) separates $F L^{P}$ and $F H^{P}$. Equating $\alpha K_{j}^{L A}=K_{j}^{L A}-(1-\hat{\sigma})$, we obtain $K_{j}^{L A^{*}}=(1-\hat{\sigma}) /(1-\alpha)$ as critical value for which (20) and (21) become binding. Thus, machine capacity $j$ is set to the sum of $K_{j}^{L A}$ * and available transfers from plant $k, \alpha K_{k}^{L A^{*}}$, where $j \neq k$. We obtain $K_{j}^{M A^{*}}=(1+\alpha)(1-\hat{\sigma}) /(1-\alpha)$.

$1 \mathrm{~b}$. If $F H^{P}$ holds, (20) and (21) are never binding. We obtain $K_{j}^{L A^{*}}=1$ and $K_{j}^{M A^{*}}=(1+\hat{\sigma})$.

2 . Next, the conditions for setting $K_{j}^{L A}>1$ are derived.

Suppose that $F L^{P}$ holds. If $\alpha<\hat{\sigma}$ and $K_{j}^{L A}>(1-\hat{\sigma}) /(1-\alpha)$, then (20) and (21) are limiting transfers. Increasing expected production by one yields $p$ and requires one unit of $K_{j}^{M A}$ and one unit of additional labor capacity (either regular workforce or transfers) in $j=1,2$. With respect to (20) and (21), the required units of additional regular workforce $\Delta K_{(\cdot)}^{L A}=1 /(1+\alpha)$ are given by $\Delta K_{(\cdot)}^{L A}+\alpha \Delta K_{(\cdot)}^{L A}=1$, where $\alpha \Delta K_{(\cdot)}^{L A}$ denotes transferred capacity (recall that $\left.\Delta K_{1}^{L A}=\Delta K_{2}^{L A}\right)$. Therefore, $\alpha /(1+\alpha)$ units of labor are transferred between $k$ and $j$ if demand in $j$ is high. Comparing 
(expected) costs with expected revenue yields

$p \geq \frac{2}{1+\alpha} c^{L A}+2 c^{M A}+\frac{\alpha}{1+\alpha} c^{t r}$.

Eq. (27)separates $P M^{P}$ and $P H^{P}$. It is beneficial to fully satisfy demand in the high demand scenario and we obtain $K_{j}^{L A^{*}}=(1+\hat{\sigma}) /(1+\alpha)$ and $K_{j}^{M A^{*}}=1+\hat{\sigma}$.

Case (iii): Since production shifting is assumed to be costless, the flexible network is equivalent to a single plant facing aggregate demand $2(1-\hat{\sigma})$ in the low demand scenario and $2(1+\hat{\sigma})$ in the high demand scenario. This implies that personnel transfers are not used since $c^{t r}>0$. We obtain the same decisions and conditions as in case (i).

Case (iv): Since production shifting is assumed to be costless, the flexible network is equivalent to a single plant facing aggregate demand of 2 in both the low and high demand scenario. Since we assume that $p>c^{L A}+c^{M A}, \sum_{j=1}^{2} K_{j}^{L A^{*}}=\sum_{j=1}^{2} K_{j}^{M A^{*}}=2$.

\section{References}

Aksin, O.Z., Karaesmen, F., Ormeci, E.L., 2005. On the interaction between resource flexibility and flexibility structures. In: Proceedings of the Fifth International Conference on Analysis of Manufacturing Systems-Production Management, Zakynthos, Greece, May 2005.

Askar, G., Sillekens, T., Suhl, L., Zimmermann, J., 2007. Flexibility planning in automotive plants. In: Günther, H.-O., Mattfeld, D.C., Suhl, L. (Eds.), Management Logistischer Netzwerke. Physica, Heidelberg, pp. 235-255.

Barkholz, D., 2009. Flexibility helps BMW's U.S. plant avoid layoff. Automotive News Europe, June 12.

Bertrand, J., 2003. Supply chain design: flexibility considerations. In: De Kok, A., Graves, S. (Eds.), Supply Chain Management: Design, Coordination and Operation, Handbooks in Operations Research and Management Science, vol. 11. Elsevier, North-Holland, Amsterdam, pp. 133-198.

Bradley, S.P., Hax, A.C., Magnanti, T.L., 1977. Applied Mathematical Programming. Addison-Wesley, Reading.

Chandra, C., Everson, M., Grabis, J., 2005. Evaluation of enterprise-level benefits of manufacturing flexibility. Omega 33 (1), 17-31.

Chod, J., Rudi, N., Van Mieghem, J.A., 2007. Mix, time, and volume flexibility: valuation and corporate diversification. Working paper.
Daimler, 2005. Sustainability Report 2005 〈http://www.daimler.com/Projects/ c2c/channel/documents/1688145_daimler_sust_2005_reports_sustainabilityr eport2005facts_en.pdf $>$.

De Saint-Seine, S., 2007. Renault steers away from one-platform, one-model plant. Automotive News Europe, June 11.

Eppen, G.D., 1979. Effects of centralization on expected costs in a multi-location newsboy problem. Management Science 25 (5), 498-501.

Fine, C., Freund, R., 1990. Optimal investment in product-flexible manufacturing capacity. Management Science 36 (4), 449-466.

Fleischmann, B., Ferber, S., Henrich, P., 2006. Strategic planning of BMW's global production network. Interfaces 36 (3), 194-208.

Francas, D., Kremer, M., Minner, S., Friese, M., 2009. Strategic process flexibility under lifecycle demand. International Journal of Production Economics 121 (2), 427-440.

Gerwin, D., 1993. Manufacturing flexibility: a strategic perspective. Management Science 39 (4), 395-410.

Goyal, M., Netessine, S., 2009. Volume flexibility with multiple products and the trade-off with product flexibility. Working paper.

Jack, E., Raturi, A., 2002. Sources of volume flexibility and their impact on performance. Journal of Operations Management 20 (5), 519-548.

Jordan, W., Graves, S., 1995. Principles on the benefits of manufacturing process flexibility. Management Science 41 (4), 577-594.

Lu, L.X. Van Mieghem, J.A., 2009. Multi-market facility network design with offshoring applications. Manufacturing \& Service Operations Management 11 (1), 90-108.

Koste, L., Malhotra, M., 1999. A theoretical framework for analyzing the dimensions of manufacturing flexibility. Journal of Operations Management 18 (1), 75-93.

Kulkarni, S., Magazine, M.J., Raturi, A.S., 2005. On the trade-offs between riskpooling and logistics costs in a multi-plant network with commonality. IIE Transactions 37 (3), 247-265.

Pinker, E.J., Larson, R.C., 2003. Optimizing the use of contingent labor when demand is uncertain. European Journal of Operational Research 144 (1), 39-55.

Suarez, F.F., Cusumano, M.A., Fine, C.H., 1996. An empirical study of manufacturing flexibility in printed circuit board assembly. Operations Research 44 (1), 223 240.

Sydsæter, K., Hammond, P., 2002. Essential Mathematics for Economic Analysis, second ed. Prentice-Hall, New Jersey.

Van Mieghem, J.A., 1998. Investment strategies for flexible resources. Managemen Science 44 (8), 1071-1078.

Van Mieghem, J.A., 2004. Note: Commonality strategies: value drivers and equivalence with flexible capacity and inventory substitution. Management Science 50 (3), 419-424.

Van Mieghem, J.A., Rudi, N., 2002. Newsvendor networks: inventory management and capacity investment with discretionary activities. Manufacturing \& Service Operations Management 4 (4), 313-335.

Vokurka, R.J., O'Leary-Kelly, S.W., 2000. A review of empirical research on manufacturing flexibility. Journal of Operations Management 18 (4), 485-501. 